\title{
Four glucosyltransferases, GtfJ, GtfK, GtfL and GtfM, from Streptococcus salivarius ATCC 25975
}

\author{
Christine L. Simpson, ${ }^{1} \dagger$ Norman W. H. Cheetham, ${ }^{2}$ Philip M. Giffard ${ }^{1} \ddagger$ \\ and Nicholas A. Jacques ${ }^{1}$
}

1 Institute of Dental Research, 2 Chalmers Street, Surry Hills, NSW 2010, Australia

2 School of Chemistry, The University of New South Wales, PO Box 1 , Kensington, NSW 2033, Australia

\author{
Author for correspondence: Nicholas A. Jacques. Tel: +6122820353 . Fax: +6122820368. \\ e-mail: dentres@angis.su.oz.au
}

\begin{abstract}
The four recombinant glucosyltransferases (GTFs), GtfJ, GtfK, GtfL and GtfM, that had previously been cloned from Streptococcus salivarius ATCC 25975, were individually expressed in Escherichia coli and their glucan products and kinetic properties were analysed. GtfJ was a primer-dependent GTF which synthesized an insoluble glucan composed mainly of $\alpha-(1 \rightarrow 3)$-linked glucosyl residues in the presence of dextran T-10. GtfK was primer-stimulated, and produced a linear soluble dextran without any detectable branch points both in the absence and in the presence of dextran T-10. GtfL was primerindependent and produced a mixed-linkage insoluble glucan composed of approximately equal proportions of $\alpha-(1 \rightarrow 3)$ - and $\alpha-(1 \rightarrow 6)$-linked glucosyl residues. GtfL was inhibited by dextran T-10. GtfM was primer-independent and produced a soluble dextran with approximately $5 \% \alpha-(1 \rightarrow 3)$-linked glucosyl residues. GtfM was essentially unaffected by the presence of dextran T-10. The results confirmed that each enzyme represented one of the four possible combinations of primer-dependency and product solubility and that each possessed unique biosynthetic properties. The soluble dextrans formed by GtfK and GtfM, as well as the mixed-linkage insoluble glucan formed by GtfL, were also capable of acting as primers for the primer-dependent GtfJ and the primer-stimulated GtfK. Unexpectedly, the linear dextran produced by GtfK was by far the least effective either at priming itself or at activating and priming the primer-dependent GtfJ.
\end{abstract}

Keywords: Streptococcus salivarius, glucosyltransferases, glucans, ${ }^{13} \mathrm{C}-\mathrm{NMR}$, kinetics

\section{INTRODUCTION}

Recent refined taxonomic studies have confirmed that Streptococcus salivarius is the dominant streptococcal species on the dorsum of the tongue (Milnes et al., 1993a). While early studies showed that $S$. salivarius could also be found on the tooth surface, especially during plaque development and following repeated ingestion of sucrose (Carlsson, 1969), a recent longitudinal study has shown that this species persists in larger numbers than was previously believed (Milnes et al., 1993b). S. salivarius has

†Present address: Department of Oral Biology, The Dental School, University of Newcastle upon Tyne, Framlington Place, Newcastle upon Tyne NE2 4BW, UK.

‡Present address: Centre for Molecular Biotechnology, Queensland University of Technology, GPO Box 2434, Brisbane, QLD 4001, Australia.

Abbreviation: GTF, $\alpha$-D-glucosyltransferase. been classified into the high-caries category, along with the mutans streptococci (Drucker et al., 1984), and many strains produce extracellular $\alpha$-D-glucosyltransferases (GTFs) that polymerize the glucose moiety of sucrose into $\alpha$-D-glucans. GTFs can be divided into four groups depending on whether they produce a soluble dextran [GTF-Ss, producing a primarily $\alpha$-( $1 \rightarrow 6)$-linked glucan] or an insoluble glucan (GTF-Is), and whether or not they require a primer-dextran for activity. Because of their role in plaque accumulation, streptococcal GTFs are arguably the most significant virulence factor in the initiation of dental caries on smooth enamel surfaces and most likely on root surfaces as well. Recent animal model studies with streptococci possessing inactivated $g t f$ genes confirm these findings (Munro et al., 1991; Yamashita et al., 1993).

Many studies have looked at the structure of total glucan produced by streptococci or the products of two enzyme 
Table 1. Bacterial strains, bacteriophages and phagemids

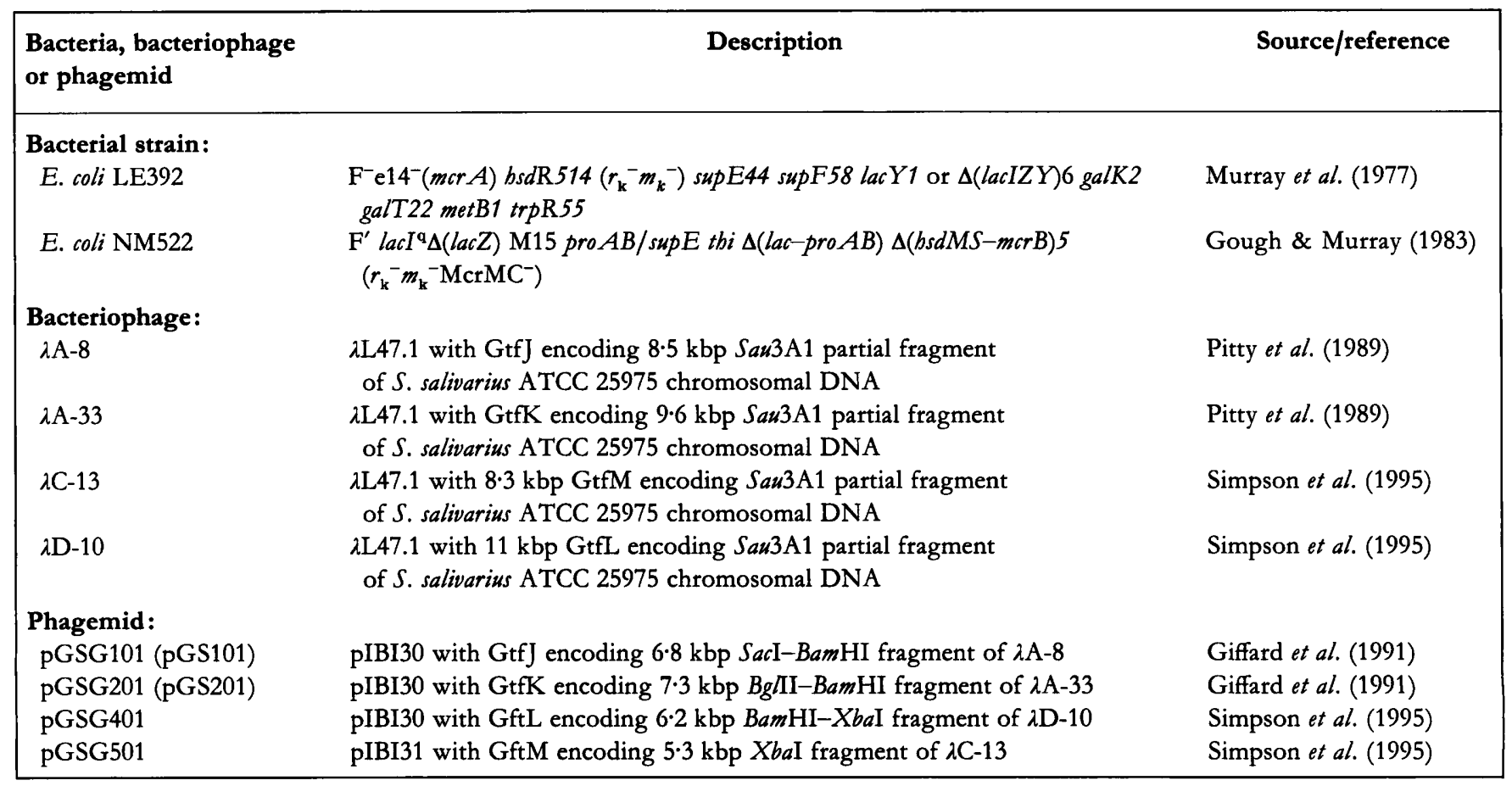

fractions, those producing water-insoluble and those producing water-soluble glucans (Walker, 1978; Walker \& Jacques, 1987). These studies have suggested that the soluble dextrans produced by the GTF-Ss act as primers for the GTF-Is. The GTF-Is synthesize chains of $\alpha-(1 \rightarrow$ $3)$-linked glucosyl residues onto the $\alpha$ - $(1 \rightarrow 6)$-linked backbone of dextrans to form insoluble mixed-linkage glucans. The insolubility of these glucans is due to the $\alpha$ $(1 \rightarrow 3)$-linked glucosyl residues as well as the degree of branching of the final glucan product (Walker, 1978; Walker \& Jacques, 1987; Takehara et al., 1992).

More recent work has indicated that many oral streptococci produce more than two GTFs. For example, two groups working on Streptococcus sobrinus have successfully separated four GTF activities from this species (Fukui $e t$ al., 1982; Shimamura et al., 1982; Hanada \& Takehara, 1987a, b, 1991; Yamashita et al., 1988a, b, 1989; Taylor et al., 1990; Walker et al., 1990; Hanada et al., 1993). The primer-dependent GTF-I produces a glucan with mainly $\alpha$-(1 $\rightarrow 3)$-linked glucosyl residues, while the other three activities produce different water-soluble products with a common $\alpha$-(1 $1 \rightarrow 6)$-linked glucosyl backbone.

The GTFs of other oral streptococci have not been as well characterized, though the cloning of individual gtf genes has allowed some structural determination of the glucans synthesized by specific GTFs (Russell et al., 1987, 1990; Nakano \& Kuramitsu, 1992). In the case of $S$. salivarius ATCC 25975, four GTFs, GtfJ, GtfK, GtfL and GtfM, have been cloned and sequenced (Giffard et al., 1991, 1993; Simpson et al., 1995). As little is known about the glucans synthesized by this species, or the enzymes that synthesize them, the availability of four different cloned
GTFs represented a unique opportunity to study some of their individual properties.

\section{METHODS}

Bacterial strains and growth conditions. Eschericbia coli strains LE392 and NM522 (Table 1) were used throughout these studies. E. coli strains were grown in Luria-Bertani (LB) medium at $37^{\circ} \mathrm{C}$ (Miller, 1972), supplemented with $100 \mu \mathrm{g}$ ampicillin $\mathrm{ml}^{-1}$ where appropriate.

Chemicals and enzymes. All chemicals were purchased from Sigma, BDH or Ajax and were analytical reagent grade or equivalent. [U- $\left.{ }^{14} \mathrm{C}\right]$ glucosyl-labelled sucrose was purchased from NEN (Dupont).

Detection of GTF activity. Total GTF activity, in the presence or the absence of $200 \mu \mathrm{g}$ dextran T-10 $\mathrm{ml}^{-1}$, was quantified using [U- ${ }^{14} \mathrm{C}$ ]glucosyl-labelled sucrose as previously described by Jacques (1983), except for time-course experiments, where the total volume was increased to $24 \mathrm{ml}$ and $1 \mathrm{ml}$ aliquots were taken in duplicate at various intervals over a 4-6h period, and the mean GTF activity of each sample (differing by $\leqslant 7 \%$ ) was calculated. The amount of insoluble glucan formed was determined by replacing the ethanol precipitation and washing steps in the above assay with washes with $10 \mathrm{mM}$ potassium phosphate buffer $(\mathrm{pH} 6.0)$. The amount of glucan formed was defined as $\mu \mathrm{mol}$ glucose incorporated into glucan per $\mathrm{ml}$ assay mixture. Shown in each case are representative results from experiments that were repeated at least once.

Preparation of recombinant GTF activities for kinetic studies. E. coli NM522, transformed with a phagemid expressing a specific GTF activity (pGSG101, pGSG201, pGSG401 or pGSG501; Table 1), was grown at $37^{\circ} \mathrm{C}$ for $16 \mathrm{~h}$ in a shaking incubator in $200 \mathrm{ml} \mathrm{LB}$ medium supplemented with $100 \mu \mathrm{g}$ ampicillin $\mathrm{ml}^{-1}$. Cells were harvested by centrifugation $(5000 \mathrm{~g}$, 


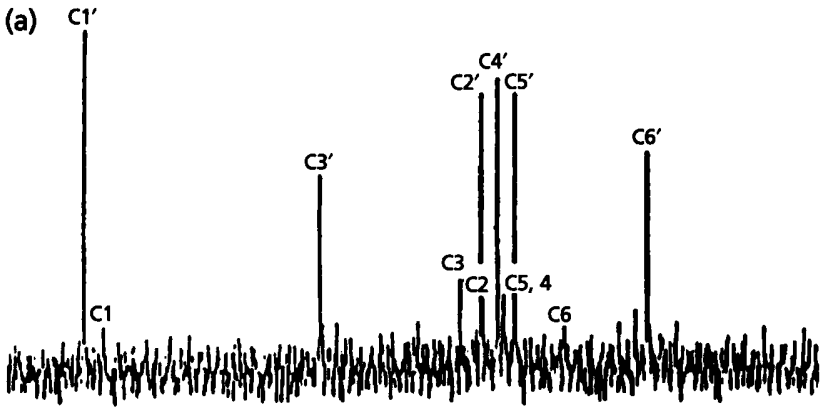

(b)

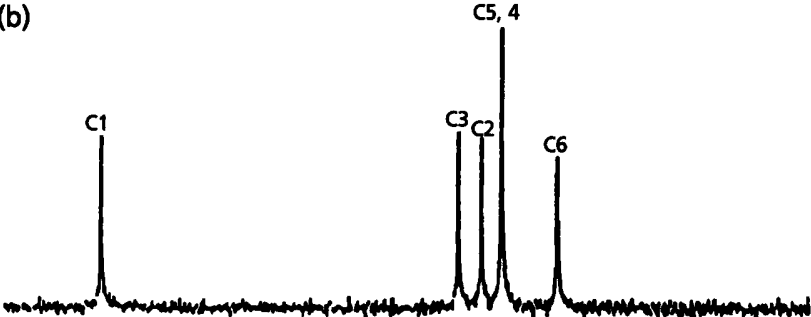

(c)

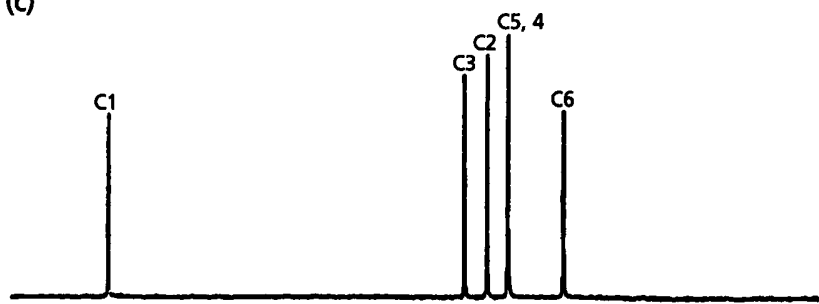

(d)

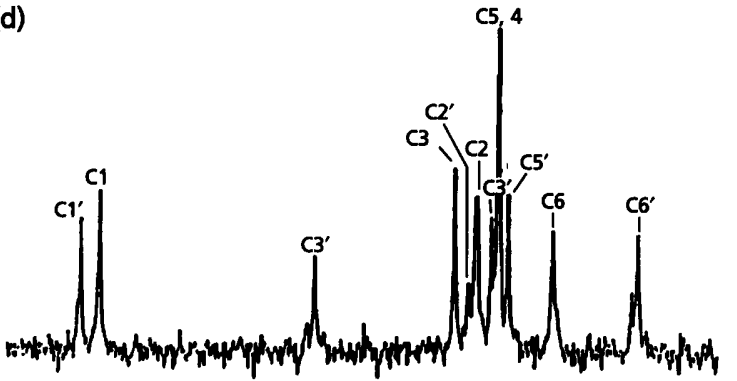

(e)

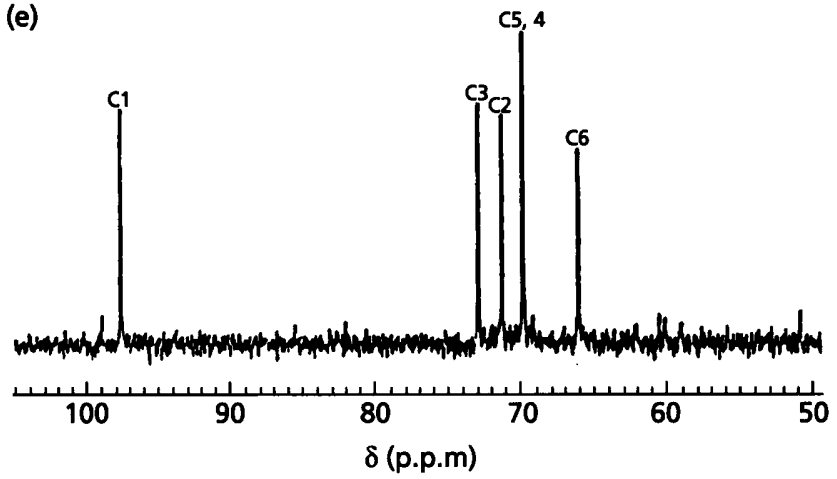

Fig. 1. ${ }^{13} \mathrm{C}$-NMR spectra of the glucans produced by the primerdependent GtfJ in the presence of $200 \mu \mathrm{g}$ dextran T-10 $\mathrm{ml}^{-1}$ (a) the 'primer-stimulated' GtfK in the absence of dextran T-10 (b), the 'primer-stimulated' GtfK in the presence of $200 \mu \mathrm{g}$ dextran $\mathrm{T}-10 \mathrm{ml}^{-1}$ (c), the primer-independent GtfL (d) and the primer-
$15 \mathrm{~min}, 4^{\circ} \mathrm{C}$ ) and resuspended in $50 \mathrm{ml} 20 \mathrm{mM}$ potassium phosphate buffer ( $\mathrm{pH} 6.5)$. The cells were sonicated on ice using a Branson Sonic Power ( $40 \mathrm{~W}, 60 \mathrm{~s}$ with 5-10 min cooling periods, for a total sonication time of $5 \mathrm{~min}$ ) and unbroken cells and cell debris were removed by centrifugation $(10000 \mathrm{~g}$, $15 \mathrm{~min}, 4^{\circ} \mathrm{C}$ ). The supernatant was used as the source of GTF activity.

Glucan products as acceptors for primer-dependent activities. Non-radiolabelled glucan was produced by incubating GtfK, GtfL or $\mathrm{GtfM}$ at $37^{\circ} \mathrm{C}$ for $2-6 \mathrm{~h}$ in $48 \mathrm{ml}$ assay mix (Jacques, 1983; see above) in the absence of added dextran T-10. Control assays containing radiolabelled sucrose were run in parallel to determine the amount of glucan produced in each instance $\left(43 \pm 5 \mu \mathrm{g} \mathrm{ml}^{-1}\right.$; range $\left.39-49 \mu \mathrm{g} \mathrm{ml}^{-1}\right)$. After incubation, the samples were boiled for $10 \mathrm{~min}$ to inactivate GTF activity. After cooling to $37^{\circ} \mathrm{C}, \mathrm{GtfJ}$ or $\mathrm{GtfK}$ was added to the experimental tubes, together with $22 \mathrm{MBq}\left[\mathrm{U}-{ }^{14} \mathrm{C}\right]$ glucosyllabelled sucrose, and the incubation was continued at $37^{\circ} \mathrm{C}$ for $4 \mathrm{~h}$. Duplicate $1 \mathrm{ml}$ aliquots were assayed at various time intervals during this period to determine both insoluble and total glucan production.

Partial purification of recombinant GTFs for glucan production. GTFs were partially purified on hydroxylapatite at $4{ }^{\circ} \mathrm{C}$ essentially as described by Walker et al. (1990). A 21 lysate was prepared as previously described by Giffard et al. (1991) from bacteriophages $\lambda \mathrm{A}-8, \lambda \mathrm{A}-33, \lambda \mathrm{D}-10$ and $\lambda \mathrm{C}-13$ encoding GtfJ, GtfK, GtfL and GtfM respectively (Table 1). Following centrifugation $\left(5000 \mathrm{~g}, 15 \mathrm{~min}, 4^{\circ} \mathrm{C}\right)$, the supernatant was concentrated 4-15-fold using a hollow-fibre system (Amicon), and sodium azide was added to $0.05 \%(\mathrm{w} / \mathrm{v}$ ) (all subsequent solutions also contained $0.05 \%(\mathrm{w} / \mathrm{v})$ sodium azide). The concentrated supernatant was dialysed at $4{ }^{\circ} \mathrm{C}$ overnight against $10 \mathrm{mM}$ potassium phosphate buffer $(\mathrm{pH} \mathrm{6.5)}$, centrifuged $\left(5000 \mathrm{~g}, 15 \mathrm{~min}, 4^{\circ} \mathrm{C}\right)$ to remove particulate matter and then applied to a column $(27 \mathrm{~cm} \times 1.6 \mathrm{~cm})$ of hydroxylapatite (BioGel HTP) prepared in the same buffer. The column was washed with 3 vols buffer and $4 \mathrm{ml}$ fractions were collected by eluting the column with a linear gradient of potassium phosphate buffer $(10-300 \mathrm{mM}, \mathrm{pH} 6 \cdot 5)$ at a flow rate of $8 \mathrm{ml} \mathrm{h}^{-1}$. Each fraction was assayed, and those under a peak containing GTF activity were pooled and dialysed against $10 \mathrm{mM}$ potassium phosphate buffer ( $\mathrm{pH} \mathrm{6.5)}$ ) at $4{ }^{\circ} \mathrm{C}$ overnight. This material was used as the source of enzyme for glucan synthesis.

Preparation of glucans. The partially purified primer-independent GtfL or GtfM, or the primer-dependent GtfJ in the presence of $200 \mu \mathrm{g}$ dextran $\mathrm{T}-10 \mathrm{ml}^{-1}$, or the "primerstimulated' GtfK in the presence of $200 \mu \mathrm{g}$ dextran T-10 $\mathrm{ml}^{-1}$, were incubated overnight at $37^{\circ} \mathrm{C}$ in $200 \mathrm{ml} 10 \mathrm{mM}$ potassium phosphate buffer, $\mathrm{pH} 6.5$, containing $4 \%(\mathrm{w} / \mathrm{v})$ sucrose and $0.01 \%(\mathrm{w} / \mathrm{v})$ thiomersal, while the 'primer-stimulated' GtfK in the absence of dextran T-10 was incubated for $5 \mathrm{~d}$. The insoluble glucans produced by $\mathrm{GtfJ}$ and $\mathrm{GtfL}$ were recovered by centrifugation $\left(10000 \mathrm{~g}, 15 \mathrm{~min}, 4^{\circ} \mathrm{C}\right)$, and resuspended in $200 \mathrm{ml} \mathrm{H} \mathrm{H}_{2} \mathrm{O}$. The latter process was repeated three times before the glucans were freeze-dried. The soluble glucans produced by $\mathrm{GtfK}$ and GtfM were precipitated by the addition of 3 vols ethanol at $4{ }^{\circ} \mathrm{C}$ and recovered by centrifugation $(10000 \mathrm{~g}$, $15 \mathrm{~min}, 4^{\circ} \mathrm{C}$ ). The glucans were dissolved in $\mathrm{H}_{2} \mathrm{O}$ and the

independent GtfM (e). The six carbons of $\alpha$-(1 $1 \rightarrow 6)$-linked glucosyl residues $(\mathrm{C} 1-\mathrm{C} 6)$ and the six carbons of $\alpha$-(1 $1 \rightarrow 3)$-linked glucosyl residues $\left(\mathrm{C1}^{\prime}-\mathrm{C6}^{\prime}\right)$ are shown. 
precipitation step was repeated three times. The glucans were then resuspended in a small volume of $\mathrm{H}_{2} \mathrm{O}$ and freeze-dried.

${ }^{13} \mathrm{C}-\mathrm{NMR}$ analysis of glucans. ${ }^{13} \mathrm{C}$-NMR spectra of saturated solutions of each glucan dissolved in deuterated DMSO were obtained at $124 \mathrm{MHz}$ in a $5 \mathrm{~mm}$ diameter sample tube, using a Bruker AM500 spectrometer operating in the Fourier-transform mode with complete proton decoupling; probe temperature was $90^{\circ} \mathrm{C}$. Spectra were run overnight accumulating 16384 data points over a spectral width of $27.7 \mathrm{kHz}$ from 26000 scans. Digital resolution was $3.39 \mathrm{~Hz}$ per point. The internal reference was DMSO (40 p.p.m.). Assignment of peaks was based on reports by Colson et al. (1974) and Gorin (1981).

\section{RESULTS AND DISCUSSION}

\section{${ }^{13} \mathrm{C}-\mathrm{NMR}$ analysis of the glucans produced by $S$. salivarius GTFs}

${ }^{13} \mathrm{C}$-NMR spectroscopy allowed the determination of the major linkages within the glucans produced by the cloned GTFs of $S$. salivarius that had been partially purified from bacteriophage $\lambda$ lysates by chromatography on hydroxylapatite. Since the percentage of linkages in carbohydrate polymers cannot be accurately quantified by ${ }^{13} \mathrm{C}-\mathrm{NMR}$ spectroscopy, the values given are only an estimate.

The glucan produced by GtfJ in the presence of $200 \mu \mathrm{g}$ dextran T-10 $\mathrm{ml}^{-1}$ was insoluble and composed mainly of $\alpha$-(1 $\rightarrow 3)$-linked glucosyl residues as indicated by the assignment of the six carbons to major peaks in the ${ }^{13} \mathrm{C}$ NMR spectrum (peaks $\mathrm{C1}^{\prime}-\mathrm{C6}^{\prime}$; Fig. 1a). The peaks marked C1-C6 (Fig. 1a) were those of $\alpha$-(1 $1 \rightarrow 6)$-linked glucosyl residues, which made up $10-15 \%$ of the total glucan. The water-insoluble glucan produced by GtfJ in the presence of dextran T-10 was therefore similar to that produced by other primer-dependent GTF-Is. For instance, the GTF-I of $S$. sobrinus 6714 produces a glucan composed of $93 \% \quad \alpha-(1 \rightarrow 3)$-linked glucosyl residues (Fukui et al., 1982), while that produced by the GTF-I of S. sobrinus AHT is composed of $79 \% \alpha-(1 \rightarrow 3)$-linked glucosyl residues (Hanada \& Takehara, 1987b). The presence of $\alpha-(1 \rightarrow 6)$-linked glucosyl residues in these polymers may have arisen from the dextran primers, for where polymers can be synthesized by GTF-I enzymes in the absence of primer dextran [such as the GtfI of Streptococcus downei expressed in E. coli (Russell et al., 1987) and the GtfI of S. sobrinus (Shimamura et al., 1983; Shimamura, 1989)], these glucans consist of essentially $100 \% \alpha$ - $(1 \rightarrow 3)$-linked glucosyl residues.

The soluble glucans produced by GtfK in the absence (Fig. 1b) or the presence (Fig. 1c) of dextran T-10 were composed of $100 \% \alpha-(1 \rightarrow 6)$-linked glucosyl residues, with no detectable branch points. The $\alpha-(1 \rightarrow 6)$-linked structure of the glucan produced by GtfK was in contrast to the glucan produced by the primer-dependent GTF-S of $S$. sobrinus, which contains $32 \% \alpha$ - $(1 \rightarrow 3)$-branch points (McCabe, 1985; Taylor et al., 1990). A primer-dependent GTF-S, GtfD, has also been cloned from Streptococcus mutans (Hanada \& Kuramitsu, 1989). However, the structure of the glucan it synthesizes has not been reported.

The insoluble glucan produced by the primer-independent GtfL contained both $\alpha-(1 \rightarrow 6)$-linked (peaks C1-C6, Fig. 1d) and $\alpha$-(1 $\rightarrow 3$ )-linked glucosyl residues (peaks $\mathrm{C1}^{\prime}-\mathrm{C}^{\prime}$, Fig. 1d). These linkages appeared to be present in roughly equal proportions. The minor resonance at 61.09 p.p.m. was consistent with an $\alpha$-(1 $1 \rightarrow 3)$-branch point. The structure of the glucan produced by GtfL was therefore analogous to that synthesized by a primerindependent GTF-I purified from another human isolate of $S$. salivarius, as this latter glucan also contains a similar percentage of both $\alpha-(1 \rightarrow 6)$ - and $\alpha-(1 \rightarrow 3)$-linked glucosyl residues (Sato \& Inoue, 1991). These enzymes from $S$. salivarius are similar to ones isolated from $S$. sobrinus which also produce mixed-linkage glucans (Cheetham et al., 1991; Hanada et al., 1993). Prior to their discovery, such a product was considered to be synthesized only as a result of the concerted action of a mixture of GTF-S and GTF-Is (Walker, 1978; Walker \& Jacques, 1987). The glucan produced by GtfL is also similar to that synthesized by a single point, site-directed mutant (T589E) of the primer-dependent GtfD of $S$. mutans. In the absence of primer, this mutant GTF has the

Table 2. Properties of the GTFs of S. salivarius ATCC 25975

\begin{tabular}{|c|c|c|c|c|c|c|c|}
\hline \multirow[t]{2}{*}{ GTF } & \multirow[t]{2}{*}{$10^{-3} \times M_{\mathrm{r}}$} & \multirow[t]{2}{*}{$\mathrm{pI}^{*}$} & \multicolumn{2}{|c|}{$K_{\mathrm{m}}(\mathrm{mM}) \dagger$} & \multirow[t]{2}{*}{ Type } & \multirow[t]{2}{*}{ Glucan $§$} & \multirow[t]{2}{*}{ Primer $\|$} \\
\hline & & & $-T-10 \ddagger$ & $+T-10 \ddagger$ & & & \\
\hline GtfJ & 168 & $5 \cdot 1$ & - & 20 & Primer-dependent & $90 \% \alpha-(1 \rightarrow 3)-$ (insoluble) & $\mathrm{T}-10 \ddagger, \mathrm{M} \gg \mathrm{L} \gg \mathrm{K}$ \\
\hline GtfK & 176 & $5 \cdot 5$ & 7 & 8 & 'Primer stimulated' & $100 \% \alpha-(1 \rightarrow 6)-($ soluble $)$ & $\begin{array}{l}\mathrm{T}-10 \neq>\mathrm{M} \gg \mathrm{L} . \\
\text { Not } \mathrm{K}\end{array}$ \\
\hline GtfL & 157 & $5 \cdot 2$ & 17 & 14 & Primer-independent & $50 \% \alpha-(1 \rightarrow 6)-/ 50 \% \alpha-(1 \rightarrow 3)-($ insoluble) & Inhibited by $T-10$ \\
\hline GtfM & 171 & 6.8 & 7 & 7 & Primer-independent & $95 \% \alpha-(1 \rightarrow 6)-$ (soluble) & - \\
\hline
\end{tabular}

* Calculated from the deduced amino acid sequence.

† Determined with sucrose at $\mathrm{pH} 6.0$.

$\mp$ T-10, dextran T-10.

§For Gtf J and GtfK, the glucan was synthesized in the presence of dextran T-10 $\left(200 \mu \mathrm{g} \mathrm{ml}^{-1}\right)$ which contains $<5 \% \alpha$-( $\left.1 \rightarrow 3\right)$-branch points.

$\| \mathrm{K}, \mathrm{L}$ and $\mathrm{M}$, glucans produced by GtfK, GtfL and GtfM, respectively. 
capacity to produce a highly branched insoluble glucan that is also composed of roughly equal proportions of $\alpha$ $(1 \rightarrow 6)$ - and $\alpha$ - $(1 \rightarrow 3)$-linked glucosyl residues (Shimamura et al., 1994).

The soluble glucan produced by the primer-independent GtfM was essentially composed of $\alpha$ - $(1 \rightarrow 6)$-linked glucosyl residues (peaks C1-C6; Fig. 1e). The minor resonances in the ${ }^{13} \mathrm{C}-\mathrm{NMR}$ spectrum did not match those of $\alpha-(1 \rightarrow 3)$-linked glucosyl residues and were not assigned. The glucans formed by the primer-independent GTF-Ss of $S$. sobrinus and $S$. downei are also primarily composed of $\alpha$ - $(1 \rightarrow 6)$-linked glucosyl residues, with little or no branching. In each species there is a primerindependent GTF-S that produces linear oligo-isomaltosaccharides (Yamashita et al., 1988a; Taylor et al., 1990; Russell et al., 1990), while $S$. sobrinus also secretes a primerindependent GTF-S that synthesizes a high- $M_{\mathbf{r}} \alpha-(1 \rightarrow 6)-$ linked glucan with $9 \%$ branch points (Taylor et al., 1990). The size of the glucans produced by the GTFs of $S$. salivarius are currently unknown, though the nature of the ${ }^{13} \mathrm{C}-\mathrm{NMR}$ spectrum would preclude the glucan produced by GtfM being composed of oligo-isomaltosaccharides.

Previous studies have suggested the presence of significant $\alpha$ - $(1 \rightarrow 4)$-linked glucosyl residues in the glucans from S. salivarius (Eifuku et al., 1989; Sato \& Inoue, 1991). The glucans produced by the four recombinant GTFs of $S$. salivarius ATCC 25975, however, do not contain any such linkages (summarized in Table 2). This may indicate that there are further uncloned GTFs produced by $S$. salivarius.

\section{General properties of GTFs expressed in E. coli}

Preliminary experiments showed that the glucans produced by the individual GTFs of $S$. salivarius expressed in $E$. coli were either $100 \%$ soluble or $100 \%$ insoluble. There was no evidence for any soluble glucan intermediates produced by the GTF-Is, GtfJ or GtfL, nor for the production of insoluble glucans by the GTF-Ss, GtfK or GtfM, whether or not dextran T-10 was required for activity (data not shown). Consequently, the glucans synthesized by Gtf J or GtfL were entrapped on glass-fibre disks and washed with phosphate buffer, while those of GtfK or GtfM were precipitated and washed in $75 \%$ $(\mathrm{v} / \mathrm{v})$ ethanol.

All four GTFs reacted differently to the presence of primer dextran. GtfJ was primer-dependent and thus was similar to previously reported primer-dependent GTF-Is that are unable to produce glucans without the addition of exogenous primer (Fukui et al., 1982; Takehara et al., 1984; Fig. 2a). GtfK, however, appeared to be similar to the GTFP3 of $S$. sobrinus and the GtfD of $S$. mutans (Hanada \& Takehara, 1991; Hanada \& Kuramitsu, 1989). Although these activities have been classified as primerdependent, they can still produce glucan without the addition of primer. We have therefore coined the term 'primer-stimulated' to describe these enzymes. As glucan production by GtfK in the absence of dextran $T-10$ appeared to be linear (Fig. 2b), this suggested that GtfK was not stimulated by its own product.
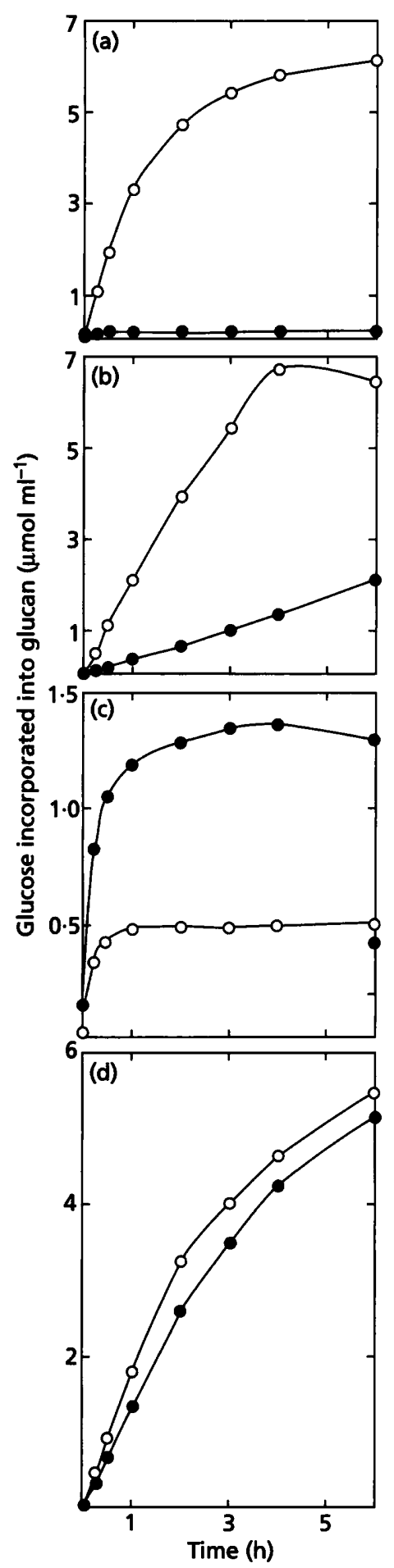

Fig. 2. Glucan production by the recombinant GTFs GtfJ (a), GtfK (b), GtfL (c) and GtfM (d), in the presence (O) or the absence $(O)$ of $200 \mu \mathrm{g}$ dextran $\mathrm{T}-10 \mathrm{ml}^{-1}$.

Compared with the three other GTFs of S. salivarius, GtfL was unique, since the addition of dextran T-10 inhibited its activity, as did, apparently, the formation of its own glucan (Fig. 2c). As a consequence, little glucan was produced by this enzyme. Studies on a similar GTF 

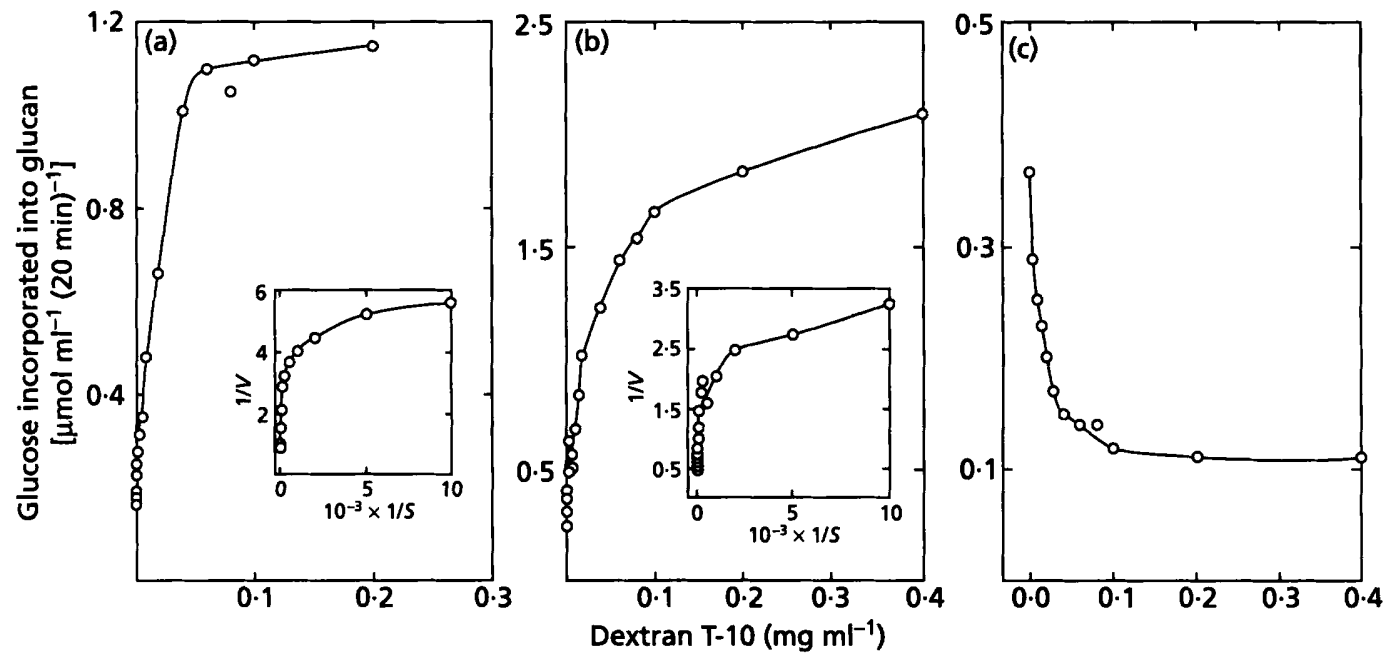

Fig. 3. Effect of dextran T-10 on glucan synthesis by the recombinant GTFs GtfJ (a), GtfK (b) and GtfL(c). Inserts show the Lineweaver-Burk plots for GtfJ (a) and GtfK (b).

isolated from S. salivarius have shown that glucan synthesis is not only inhibited by concentrations of dextran T-10 greater than $1 \mu \mathrm{g} \mathrm{ml}^{-1}$, but also by the presence of a GTF$S$ activity that presumably produces a soluble dextran (Sato \& Inoue, 1991). Previous studies on the effect of growth of oral streptococci in the presence of the surfactant Tween 80 have shown that dextran T-10 does not stimulate glucan production by the combined extracellular GTF activities of $S$. salivarius, but rather inhibits it at concentrations above $50 \mu \mathrm{g} \mathrm{ml}^{-1}$. This is the case whether or not Tween 80 is present in the growth medium (Wittenberger et al., 1978). This result differs from that obtained with $S$. sobrinus. In this instance, the combined extracellular GTF activities are stimulated by dextran T10 after growth in medium lacking Tween 80 , but not after growth in the presence of the surfactant. In the latter case, the combined GTF activities are again inhibited by concentrations of dextran T-10 greater than $50 \mu \mathrm{g} \mathrm{ml}^{-1}$ (Wittenberger et al., 1978). Recent findings may explain this phenomenon since growth of $S$. sobrinus in Tween 80 appears to promote the secretion of two otherwise undetected primer-independent GTF-Ss as well as increasing the amounts of a primer-dependent GTF-I (Walker et al., 1990). However, no specific GTFs that are inhibited by relatively low amounts of exogenous dextran have been reported from any other oral streptococcal species, other than the GtfL cloned from $S$. salivarius.

Glucan production by GtfM did not appear to be significantly stimulated by dextran T-10 (Fig. 2d). Similar results have been found for the two primer-independent GTFs of S. sobrinus, which also produce water-soluble glucans (McCabe, 1985; Yamashita et al., 1988a). This suggests that the GTF acceptor reaction is not occurring to any significant extent in these cases, and that glucan production can proceed at a near-maximum rate by autopolymerization of the glucose moiety of sucrose alone. Interestingly, the primer-independent GTF-S of $S$. sobrinus that produces a high- $M_{\mathrm{r}}$ glucan appears to bind to dextran but apparently lacks the ability to extend it (McCabe, 1985).

\section{Kinetic properties of GTFs expressed in E. coli}

The effect of increasing the concentration of dextran T-10 in the range $0-400 \mu \mathrm{g} \mathrm{ml}^{-1}$ on the initial rate of activity (0-10 $\mathrm{min}$ for GtfL due to self-inhibition, and 0-20 min for GtfJ, GtfK and GtfM) of the four S. salivarius GTFs was measured in the presence of $10 \mathrm{mM}$ sucrose at $\mathrm{pH} 6 \cdot 0$. Both GtfJ and GtfK were stimulated by increasing concentrations of dextran T-10 (Fig. 3a, b). LineweaverBurk analyses of these data were, however, non-linear (Fig. 3a, b). The steep downwards inflection at high dextran concentrations has previously been observed for a GTF activity from Streptococcus gordonii by Mayer et al. (1981), who concluded that dextran T-10 acted both as a primer and as an activator, and that the downwards inflection at high concentrations of dextran T-10 was due to the activation process. They suggested that binding to dextran T-10 might bring about an alteration in the domain surrounding the active site which results in a stimulated rate of catalysis and hence extension of the dextran T-10 primer.

In contrast to the results with GtfJ and GtfK, GtfL was strongly inhibited by exogenous dextran T-10 (see above), with maximum inhibition occurring at $100 \mu \mathrm{g}$ dextran $\mathrm{T}$ $10 \mathrm{ml}^{-1}$ (Fig. 3c). In the case of $\mathrm{GtfM}$, increasing the concentration of dextran gave rise to a slight increase in total glucan synthesis. This increase was linear over the range of dextran concentrations used and resulted in a $15 \%$ increase in apparent dextran synthesis when $400 \mu \mathrm{g}$ dextran $\mathrm{T}-10 \mathrm{ml}^{-1}$ was added to the reaction mixture (data not shown). GtfM was therefore a truly primer-independent GTF-S (cf. Fig. 2d), which appeared to be able to utilize pre-formed dextran when available.

The combined results of these studies imply that the concentration of dextran T-10 $\left(200 \mu \mathrm{g} \mathrm{ml}^{-1}\right)$ routinely 


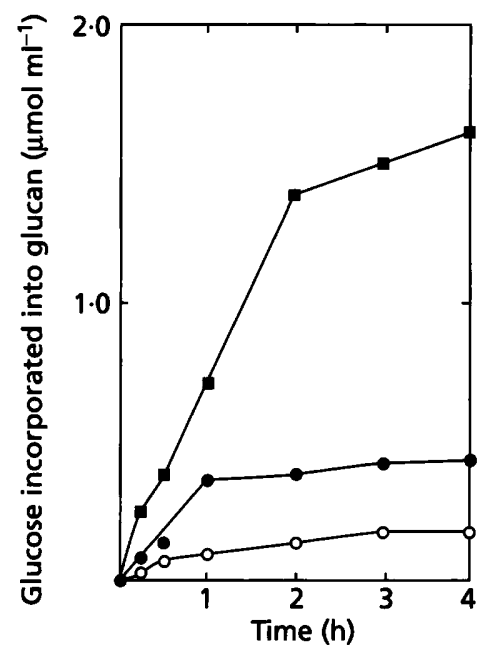

Fig. 4. Glucan synthesis by GtfJ in the presence of the glucans produced by the recombinant GTFs GtfK (O), GtfL $(O)$ and GtfM ( $\square$ ).

used to assay specific GTF activities in the presence of $10 \mathrm{mM}$ sucrose maximally inhibited GtfL, but maximally stimulated GtfJ and GtfK, and had a negligible effect on the activity of $\mathrm{GtfM}$.

The effect of increasing the concentration of sucrose in the range $0-100 \mathrm{mM}$ on the initial rates of activity was also measured in the presence or the absence of $200 \mu \mathrm{g}$ dextran T-10 $\mathrm{ml}^{-1}$ at $\mathrm{pH} 6.0$ (except for GtfJ, which did not produce glucan without primer). Each enzyme exhibited Michaelis-Menten kinetics (data not shown). Lineweaver-Burk analyses of these data enabled the $K_{\mathrm{m}}$ values for sucrose for each of the four $S$. salivarius GTFs to be determined. All fell within the range $7-20 \mathrm{mM}$ (Table 2).

\section{Acceptor efficiency of glucans produced by GtfK, GtfL and GtfM}

The glucans $\left(43 \pm 5 \mu \mathrm{g} \mathrm{ml}^{-1}\right)$ produced by GtfK, GtfL and GtfM were used as primers for GtfJ and GtfK. The amount of glucan was standardized at this concentration so that comparisons could be made, since product inhibition of GtfL prevented higher concentrations of this insoluble glucan from being made by the enzyme. At this concentration, the glucans were not expected to maximally stimulate GtfJ or GtfK (cf. Fig. 3a, b).

GtfJ was able to utilize all three glucans as acceptors for further glucan synthesis, with the dextran produced by GtfM being the most efficient primer (Fig. 4, Table 2). In each case, glucan synthesis was virtually complete within $2 \mathrm{~h}$. In the case of the dextran synthesized by GtfM, this may have been due to the formation of an insoluble product as $\alpha-(1 \rightarrow 3)$-linked glucosyl residues were added to the dextran. However, no such simple explanation could account for the limited glucan production observed when the dextran synthesized by GtfK was used as the

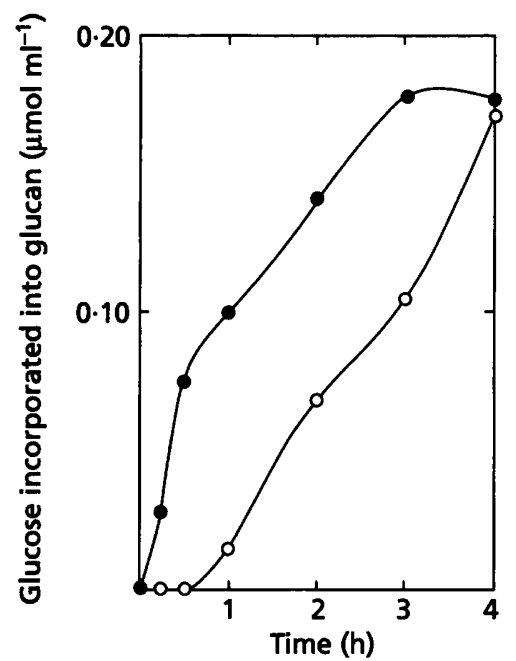

Fig. 5. Insoluble (O) and total (O) glucan produced by GtfJ in the presence of the glucan produced by GtfK.

primer. Interestingly, soluble intermediates were noted in the first $2 \mathrm{~h}$ when the dextran produced by GtfK, but only GtfK, was used as a primer for GtfJ (Fig. 5). This observation suggested that the dextran formed by GtfK was much larger than both dextran T-10 and that produced by $\mathrm{GtfM}$, as no soluble intermediates were detected when the latter two dextrans were used as primers. The ability of dextrans to act as acceptors for primer-dependent GTF-Is is dependent upon the length of unbranched $\alpha$ - $(1 \rightarrow 6)$-linked glucosyl residues within the dextrans, since it is within these regions that branch points are believed to be made, onto which new chains of $\alpha$-(1 $\rightarrow 3)$-linked glucosyl residues can be synthesized (Walker \& Schuerch, 1986). According to this model it is expected that both the dextrans produced by GtfK and GtfM should act equally efficiently as primers for the GtfJ of $S$. salivarius, since synthetic dextrans of this type have previously been reported to activate primer-dependent GTF-Is (Hare et al., 1978). This, however, was not the case, since the dextran formed by GtfM was a far better primer for the GtfJ of $S$. salivarius than that synthesized by GtfK.

Glucans synthesized by GtfM and GtfL were also capable of acting as primers for GtfK. Only soluble glucan was produced by $\mathrm{GtfK}$ when the glucan synthesized by GtfM was used as a primer (data not shown). In the reaction of $\mathrm{GtfK}$ with the glucan produced by GtfL, both soluble and insoluble glucans were detected. The amount of soluble glucan was greater than that expected through autopolymerization of the glucose moiety of sucrose by GtfK in the absence of primer, indicating that the insoluble glucan produced by GtfL was not only acting as an acceptor for GtfK but was also stimulating the synthesis of soluble glucan (Fig. 6).

The homologous glucan produced by GtfK was not selfpriming (Fig. 7, Table 2), thus confirming previous 


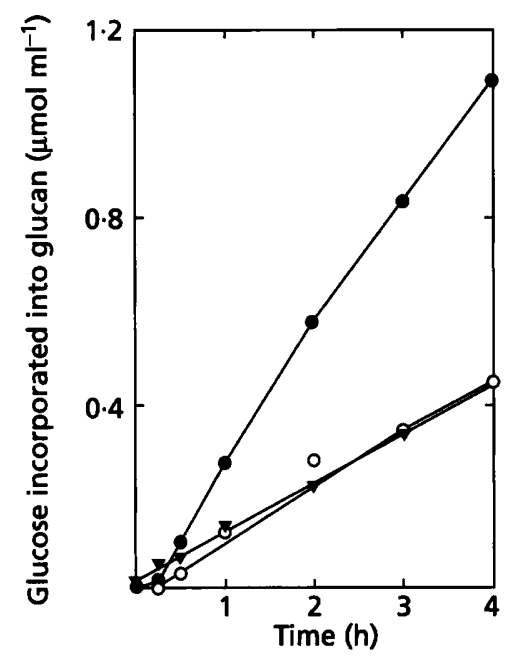

Fig. 6. Insoluble $(O)$ and total (O) glucan produced by GtfK in the presence of the glucan produced by GtfL. The total glucan (soluble) produced by GtfK in the absence of primer $(\nabla)$ is also shown.

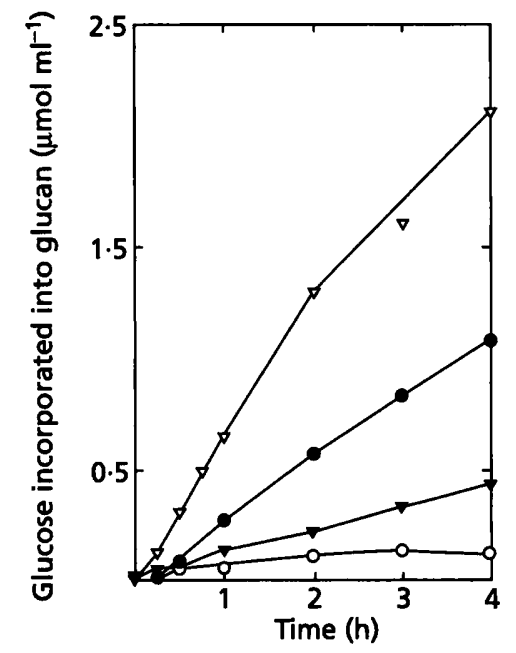

Fig. 7. Glucan synthesis by GtfK in the absence $(\nabla)$ and the presence of the glucans produced by the recombinant GTFs, GtfK (O), GtfL $(O)$ and GtfM $(\nabla)$.

observations (cf. Fig. 2b). However, as the glucan produced by GtfK was also a poor primer for GtfJ, further experiments were performed to determine whether boiling of the glucan during the assay procedure had any deleterious effect on its ability to act as an acceptor. To this end, both GtfJ and GtfK were assayed in the presence of freeze-dried glucan produced by GtfK $\left(40 \mathrm{mg} \mathrm{ml}^{-1}\right)$. The results confirmed that the glucan produced by GtfK was a poor acceptor for both enzymes (data not shown).

Although glucan synthesis by primer-dependent GTF-Is has been studied in some detail, little work has been done to determine the mechanism for glucan synthesis by primer-dependent GTF-Ss. Early experiments with a mixed GTF preparation from $S$. sobrinus indicated that $\alpha$ $(1 \rightarrow 6)$-linked glucosyl residues were formed on the nonreducing ends of isomaltosaccharides (Walker, 1973). If chain lengthening does occur in this manner then the difference between the dextran produced by GtfM and that synthesized by GtfK, itself, to act as efficient primers for $\mathrm{GtfK}$, may be due to the relative sizes of the two dextrans and thus the number of non-reducing ends available for chain-elongation.

The insoluble glucan produced by GtfL possessed approximately $50 \% \alpha-(1 \rightarrow 6)$ - and $50 \% \alpha-(1 \rightarrow 3)$-linked glucosyl residues and exhibited limited acceptor activity for both the primer-dependent GTFs, GtfJ and GtfK. This finding was unexpected as adsorption of enzymes onto insoluble glucan is generally believed to lead to their inactivation (Walker, 1978). Neither $\alpha$-(1 $1 \rightarrow 3)$-linked glucan nor the insoluble glucan produced by $S$. sobrinus can stimulate glucan synthesis by the primer-dependent GTF-S of S. sobrinus (Hare et al., 1978). Furthermore, an $\alpha-(1 \rightarrow 3)$-linked glucan, made soluble by the introduction of carboxymethyl groups, does not stimulate the GTF-I of this bacterium (Walker \& Schuerch, 1986). As the glucan synthesized by GtfL was able to act as an acceptor, this would suggest that lengths of $\alpha-(1 \rightarrow 6)$-linked glucosyl residues may remain 'dissolved' in water despite their attachment to the $\alpha$-(1 $\rightarrow 3)$-linked sequences (Walker, 1978). The fact that the glucan produced by GtfL acted as a better acceptor for GtfK than the dextran synthesized by GtfK itself is consistent with the notion that the glucan produced by GtfL is highly branched and thus has a number of non-reducing ends that are available for chain-lengthening. The increase in soluble glucan resulting from this reaction also suggests that the glucan produced by GtfL was either activating GtfK to synthesize more soluble glucan, or that the insoluble glucan itself was being 'solubilized' by the addition of $\alpha$ $(1 \rightarrow 6)$-linked glucosyl residues (Walker, 1978). Further analysis of the products of this reaction is necessary to clarify the situation.

The dextran produced by GtfM was an efficient acceptor for both GtfJ and GtfK. This raises the question as to whether the dextran synthesized by GtfM acts as a primer for $\mathrm{GtfJ}$ and/or GtfK in vivo, since in other species of oral streptococci, the glucans produced by some primerindependent GTF-Ss do not appear to be natural primers for their primer-dependent GTFs. For instance, primerindependent GTF-Ss have been reported from both $S$. sobrinus and $S$. downei (Takehara et al., 1984; McCabe, 1985; Gilpin et al., 1985; Russell et al., 1990; Taylor et al., 1990; Hanada et al., 1993), that produce either high- $M_{\mathrm{r}}$ branched dextrans (similar to the dextran synthesized by GtfM), or linear oligo-isomaltosaccharides. A mutant of $S$. downei that does not produce the primer-independent GtfS that synthesizes oligo-isomaltosaccharides from sucrose can no longer synthesize water-insoluble glucan, and has a decreased ability to adhere to glass, despite the fact that two other GTF-Ss are secreted by $S$. downei (Gilmore et al., 1993). Furthermore, while a similar enzyme to GtfS is produced by $S$. sobrinus, it does not 
synthesize an effective primer for its own primer-dependent GTF-I and GTF-S, but rather appears to reduce the molecular size of the glucan produced by the other primer-independent GTF-S of S. sobrinus (Yamashita et al., 1989).

This study indicates that the four GTFs cloned from $S$. salivarius ATCC 25975 each possess different kinetic and synthetic properties (Table 2). The results also pose the question whether a single catalytic mechanism is sufficient to describe this family of enzymes.

\section{ACKNOWLEDGEMENTS}

This work was supported by a grant from the Australian National Health and Medical Research Council.

\section{REFERENCES}

Carlsson, J. (1969). A numerical taxonomic study of human oral streptococci. Odont Revy 19, 137-159.

Cheetham, N. W. H., Walker, G. J., Pearce, B. J., Fiala-Beer, E. \& Taylor, C. (1991). Structures of water-soluble $\alpha$-D-glucans synthesized from sucrose by glucosyltransferases isolated from Streptococcus sobrinus culture filtrates. Carbobydr Polym 14, 3-16.

Colson, P., Jennings, H. J. \& Smith, I. C. P. (1974). Composition, sequence and conformation of polymers and oligomers of glucose as revealed by carbon 13 nuclear magnetic resonance. $\mathrm{J} \mathrm{Am} \mathrm{Chem}$ Soc 96, 8081-8087.

Drucker, D. B., Shakespeare, A. P. \& Green, R. M. (1984). The production of dental plaque and caries by the bacterium Streptococcus salivarius in gnotobiotic WAG/RIJ rats. Arch Oral Biol 29, 437-443.

Eifuku, H., Yoshimitsu-Narita, A., Sato, S., Yakushui, T. \& Inoue, M. (1989). Production and partial characterization of the extracellular polysaccharides from oral Streptococcus salivarius. Carbobydr Res 194, 247-260.

Fukui, K., Moriyama, T., Miyake, Y., Mizutani, K. \& Tanaka, O. (1982). Purification and properties of glucosyltransferase responsible for water-insoluble glucan synthesis from Streptococcus mutans. Infect Immun 37, 1-9.

Giffard, P. M., Simpson, C. L., Milward, C. P. \& Jacques, N. A. (1991). Molecular characterization of a cluster of at least two glucosyltransferase genes in Streptococcus salivarius ATCC 25975. J Gen Microbiol 137, 2577-2593.

Giffard, P. M., Allen, D. M., Milward, C. P., Simpson, C. L. \& Jacques, N. A. (1993). Sequence of the gtfK gene of Streptococcus salivarius ATCC 25975 and evolution of the gtf genes of oral streptococci. J Gen Microbiol 139, 1511-1522.

Gilmore, K. S., Russell, R. R. B. \& Ferretti, J. J. (1993). Expression of $g t f S$ is essential for normal insoluble glucan synthesis by Streptococcus downei. Infect Immun 61, 1246-1250.

Gilpin, M. L., Russell, R. R. B. \& Morrissey, P. (1985). Cloning and expression of two Streptococcus mutans glucosyltransferases in Eschericbia coli K12. Infect Immun 49, 414-416.

Gorin, P. J. A. (1981). Carbon-13 nuclear magnetic resonance spectroscopy of polysaccharides. Adv Carbobydr Chem Biochem 38, 13-104.

Gough, J. A. \& Murray, N. E. (1983). Sequence diversity among related genes for recognition of specific targets in DNA molecules. J Mol Biol 166, 1-19.

Hanada, N. \& Kuramitsu, H. K. (1989). Isolation and charac- terization of the Streptococcus mutans gtfD gene, coding for primerdependent soluble glucan synthesis. Infect Immun 57, 2079-2085.

Hanada, N. \& Takehara, T. (1987a). Comparison of different watersoluble glucan synthases from Streptococcus mutans serotype $g$. Microbios 50, 147-152.

Hanada, N. \& Takehara, T. (1987b). (1 $\rightarrow 3)-\alpha$-D-glucan synthase from Streptococcus mutans AHT (serotype $g$ ) does not synthesise glucan without primer. Carbobydr Res 168, 120-124.

Hanada, N. \& Takehara, T. (1991). Substrate specificity of hydrolase activity of the primer-dependent glucosyltransferases from Streptococcus sobrinus. Microbios 66, 21-25.

Hanada, N., Katayama, T., Kunimori, A., Yamashita, Y. \& Takehara, T. (1993). Four different types of glucans synthesised by glucosyltransferases from Streptococcus sobrinus. Microbios 73, 23-35.

Hare, M. D., Svensson, S. \& Walker, G. J. (1978). Characterization of the extracellular, water-insoluble $\alpha$-D-glucans of oral streptococci by methylation analysis, and by enzymic synthesis and degradation. Carbobydr Res 66, 254-264.

Jacques, N. A. (1983). Membrane perturbation by cerulenin modulates glucosyltransferase secretion and acetate uptake by Streptococcus salivarius. J Gen Microbiol 129, 3293-3302.

McCabe, M. M. (1985). Purification and characterization of a primer-independent glucosyltransferase from Streptococcus mutans 6715-13 mutant 27. Infect Immun 50, 771-777.

Mayer, R. M., Matthews, M. M., Futerman, C. L., Parnaik, V. K. \& Jung, S. M. (1981). Dextransucrase: acceptor substrate reactions. Arch Biochem Biophys 208, 278-287.

Miller, J. H. (1972). Experiments in Molecular Genetics. Cold Spring Harbor, NY: Cold Spring Harbor Laboratory.

Milnes, A. R., Bowden, G. H., Gates, D. \& Tate, R. (1993a). Predominant cultivable microorganisms on the tongue of preschool children. Microb Ecol Health Dis 6, 229-235.

Milnes, A. R., Bowden, G. H., Gates, D. \& Tate, R. (1993b). Normal microbiota on the teeth of preschool children. Microb Ecol Health Dis 6, 213-227.

Munro, C., Michalek, S. M. \& Macrina, F. L. (1991). Cariogenicity of Streptococcus mutans V403 glucosyltransferase and fructosyltransferase mutants constructed by allellic exchange. Infect Immun 59, 2316-2323.

Murray, N. E., Brammar, W. H. \& Murray, K. (1977). Lambdoid phages that simplify the recovery of in vitro recombinants. Mol \& Gen Genet 150, 53-61.

Nakano, Y. J. \& Kuramitsu, H. K. (1992). Mechanism of Streptococcus mutans glucosyltransferases: hybrid-enzyme analysis. J Bacteriol 174, 5639-5646.

Pitty, L. J., Giffard, P. M., Gilpin, M. L., Russell R. R. B. \& Jacques, N. A. (1989). Cloning and expression of glycosyltransferase activities from Streptococcus salivarius. J Dent Res 68, 1681-1682.

Russell, R. R. B., Gilpin, M. L., Mukasa, H. \& Dougan, G. (1987). Characterization of glucosyltransferase expressed from a Streptococcus sobrinus gene cloned in Escherichia coli. J Gen Microbiol 133, 935-944.

Russell, R. R. B., Gilpin, M. L., Hanada, N., Yamashita, Y., Shibata, Y. \& Takehara, T. (1990). Characterization of the product of the gtfS gene of Streptococcus downei, a primer-independent enzyme synthesizing oligo-isomaltosaccharides. J Gen Microbiol 136, 1631-1637.

Sato, S. \& Inoue, M. (1991). Partial characterization of the glucosyltransferases of an oral Streptococcus salivarius strain. Microbios 68, 179-188.

Shimamura, A. (1989). Use of ${ }^{13}$ C-N. M. R. spectroscopy for the 
quantitative estimation of 3-O- and 3,6-di-O-substituted Dglucopyranosyl residues in $\alpha$-D-glucans formed by the Dglucosyltransferases of Streptococcus sobrinus. Carbobydr Res 185, 239-248.

Shimamura, A., Tsumori, H. \& Mukasa, H. (1982). Purification and properties of Streptococcus mutans extracellular glucosyltransferase. Biochim Biopbys Acta 702, 72-80.

Shimamura, A., Tsumori, H. \& Mukasa, H. (1983). Three kinds of extracellular glucosyltransferases from Streptococcus mutans 6715 (serotype g). FEBS Lett 157, 79-84.

Shimamura, A., Nakano, Y. J., Mukasa, H. \& Kuramitsu, H. K. (1994). Identification of amino acid residues in Streptococcus mutans glucosyltransferases influencing the structure of the glucan product. $J$ Bacteriol 176, 4845-4850.

Simpson, C. L., Giffard, P. M. \& Jacques, N. A. (1995). Streptococcus salivarius ATCC 25975 possesses at least two genes coding for primer-independent glucosyltransferases. Infect Immun 63, 609-621.

Takehara, T., Hanada, N. \& Saeki, E. (1984). Interaction of glucosyltransferase isozymes on glucan synthesis by Streptococcus mutans AHT (serotype g). Microbios Lett 27, 113-120.

Takehara T., Ansai, T., Yamashita, Y., Itoh-Andoh, M., Hanada, N. \& Kunimori, A. (1992). Mechanism of water-insoluble glucan synthesis in Streptococcus sobrinus. Oral Microbiol Immunol 7, 155-158.

Taylor, C., Cheetham, N. W. H., Slodki, M. E. \& Walker, G. J. (1990). Action of endo- $(1 \rightarrow 6)-\alpha$-D-glucanases on the soluble dextrans produced by three extracellular $\alpha$-D-glucosyltransferases of Streptococcus sobrinus. Carbobydr Polymers 13, 423- 436.

Walker, G. J. (1973). Preparation of isomaltose oligosaccharides labelled with ${ }^{14} \mathrm{C}$ in the non-reducing terminal unit, and their use in studies of dextranase activity. J Dent Res 52, 1-10.

Walker, G. J. (1978). Dextrans. Int Rev Biochem 16, 75-126.
Walker, G. J. \& Jacques, N. A. (1987). Polysaccharides of oral streptococci. In Sugar Transport and Metabolism in Gram-positive Bacteria, pp. 39-68. Edited by J. Reizer \& A. Peterkofsky. Chichester: Ellis Horwood.

Walker, G. J. \& Schuerch, C. (1986). Activity of branched dextrans in the acceptor reaction of a glucosyltransferase (GTF-I) from Streptococcus mutans OMZ176. Carbobydr Res 146, 259-270.

Walker, G. J., Cheetham, N. W. H., Taylor, C., Pearce, B. J. \& Slodki, M. E. (1990). Productivity of four $\alpha$-D-glucosyltransferases released by Streptococcus sobrinus under defined conditions in continuous culture. Carbobydr Polym 13, 399-421.

Wittenberger, C. L., Beaman, A. J. \& Lee, L. N. (1978). Tween 80 effect on glucosyltransferase synthesis by Streptococcus salivarius. J Bacteriol 133, 231-239.

Yamashita, Y., Hanada, N. \& Takehara, T. (1988a). A novel glucosyltransferase from Streptococcus mutans produces oligoisomaltosaccharides. Biochem Biopbys Res Commun 150, 687-693.

Yamashita, Y., Shigeoka, T., Hanada, N. \& Takehara, T. (1988b). Immunological properties of the primer-independent glucosyltransferase of Streptococcus mutans serotypes $d$ and $g$. J Gen Microbiol 134, 1223-1227.

Yamashita, Y., Hanada, N. \& Takehara, T. (1989). Purification of a fourth glucosyltransferase from Streptococcus sobrinus. J Bacteriol 171, 6265-6270.

Yamashita, Y., Bowen, W. H., Burne, R. A. \& Kuramitsu, H. K. (1993). Role of the Streptococcus mutans gtf genes in caries induction in the specific-pathogen-free rat model. Infect Immun 61, 3811-3817.

Received 24 November 1994; revised 7 February 1995; accepted 17 February 1995. 